\title{
Chronic peripheral edema in a patient with rheumatoid arthritis
}

\author{
Vesna Breznik $^{1 凶}$, Klara Dai ${ }^{1}$, Maruška Marovt ${ }^{1}$
}

\begin{abstract}
Chronic peripheral edema is a very common yet underestimated problem. It is of utmost importance to investigate various possible causes and understand the pathophysiological mechanisms of chronic peripheral edema in order to determine the optimal treatment. This case report presents the case of a polymorbid patient with a deformity-causing type of rheumatoid arthritis and a 1-year history of progressive symmetrical peripheral edema. Based on an extensive diagnostic investigation, the patient was diagnosed with a combined form of chronic peripheral edema comprised of true lymphedema, which was confirmed by lymphoscintigraphy, and filtration edema due to muscular inactivity. Edema as a side effect of tocilizumab was also suspected.
\end{abstract}

Keywords: chronic peripheral edema, rheumatoid arthritis, lymphedema, lymphoscintigraphy, tocilizumab, decongestive lymphatic therapy

Received: 6 September 2017 | Returned for modification: 22 October 2017 | Accepted: 19 November 2017

\section{Introduction}

Chronic peripheral edema is a very common yet underestimated problem (1). It can be symmetric or asymmetric and can result from various pathologies. Symmetric chronic peripheral edema is usually of systemic origin, most commonly right heart failure, salt and water overload, venous disease, hypoalbuminemia, limb dependency or immobility, certain drugs, or chronic inflammatory conditions such as rheumatoid arthritis (RA) (2). Asymmetric edema is more likely to result from a peripheral pathology, such as impaired local venous or lymph drainage, malignancy, trauma, or infection $(2,3)$. Pathophysiologically, all types of chronic peripheral edema arise due to an imbalance between microvascular filtration and lymph drainage. It is essential to investigate various possible causes and understand the pathophysiological mechanisms in order to determine the optimal treatment of chronic peripheral edema $(2,3)$.

\section{Case report}

A 71-year-old man presented with a 1-year history of progressive symmetric peripheral edema of both the lower and upper extremities. His edema had been unsuccessfully treated previously with diuretics.

The patient's history was significant for myocardial infarction, orthopedic surgeries of the left ankle and right knee, hypertension, dyslipidemia, and benign prostatic hyperplasia. He was dependent on a wheelchair after suffering from an aggressive and deformity-causing anti-CCP-positive and RF-positive RA for more than 30 years. In the preceding year he had been treated with the biologic drug tocilizumab. His RA was in remission. His current therapy consisted of tocilizumab, acetylsalicylic acid, metoprolol, enalapril, meloxicam, rosuvastatin, and tamsulosin. During hospitalization, necrotizing cellulitis with a consequent ulcer on the right lower leg developed. Consequently, therapy with tocilizumab was discontinued, and methylprednisolone $4 \mathrm{mg}$ daily was introduced.

A physical examination at admission revealed significant pitting edema of the toes, feet, and shins, as well as of the fingers, hands, and forearms. A positive Stemmer's sign was observed in all four extremities (Figs. 1-2).

The patient's laboratory values for erythrocyte sedimentation rate, C-reactive protein, serum albumin, electrolytes, liver, kidney, and thyroid function tests, and quick ACTH test were unremarkable. ANA (1:160) and p-ANCA tests were positive. His serum amyloid A (SAA) level was elevated at $164 \mathrm{mg} / 1$ (normal $<1.2 \mathrm{mg} / \mathrm{l}$ ). His complement levels were moderately reduced: a $\mathrm{C}_{3}$ of $0.66 \mathrm{~g} / 1$ (normal range 0.9-1.8 g/l) and a $\mathrm{C}_{4}$ of $0.09 \mathrm{~g} / \mathrm{l}$ (normal range $0.1-0.4$ $\mathrm{g} / \mathrm{l})$. A quantitative and functional analysis of C1-inhibitor, as well as C1q level, were within the normal ranges. An echocardiogram, chest X-ray, abdominal ultrasound, and Doppler ultrasound of the leg veins were unremarkable. A qualitative lymphoscintigraphy of the lower extremities revealed very slow lymphatic flow in both legs, with inguinal nodes opacifying only after 4 hours. A biopsy specimen from the dorsum of the right hand showed mild nonspecific chronic perivascular inflammatory infiltrate with no signs of amyloidosis and no fibrin deposition.

Due to frailty and poor mobility, the patient received modified intensive decongestive lymphatic therapy (DLT) consisting of non-

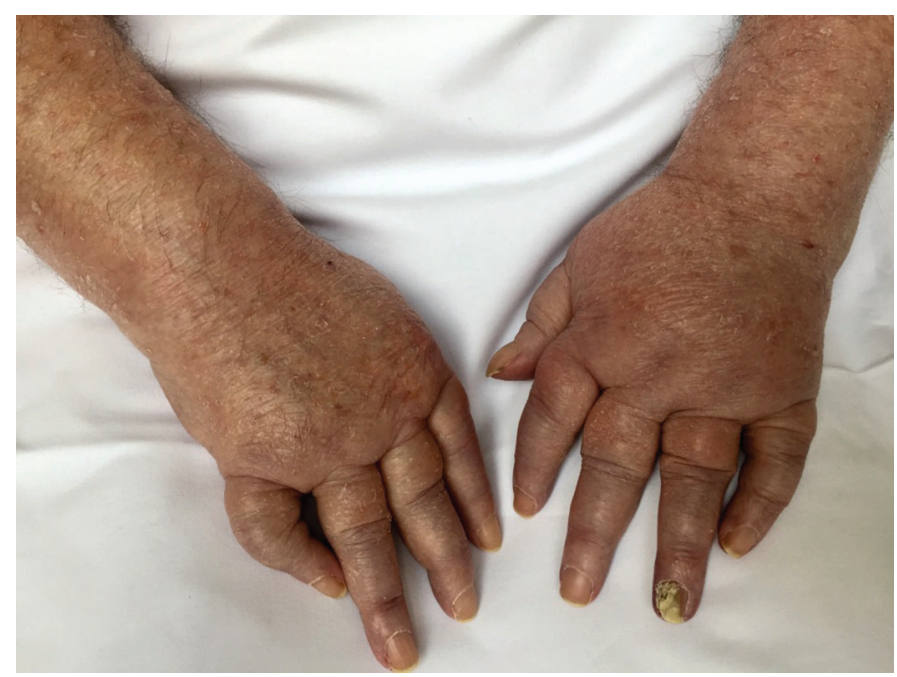

Figure 1 | Chronic edema of the upper extremities in a patient with a deformitycausing type of rheumatoid arthritis. The edema appeared after the introduction of treatment with the biologic drug tocilizumab. 


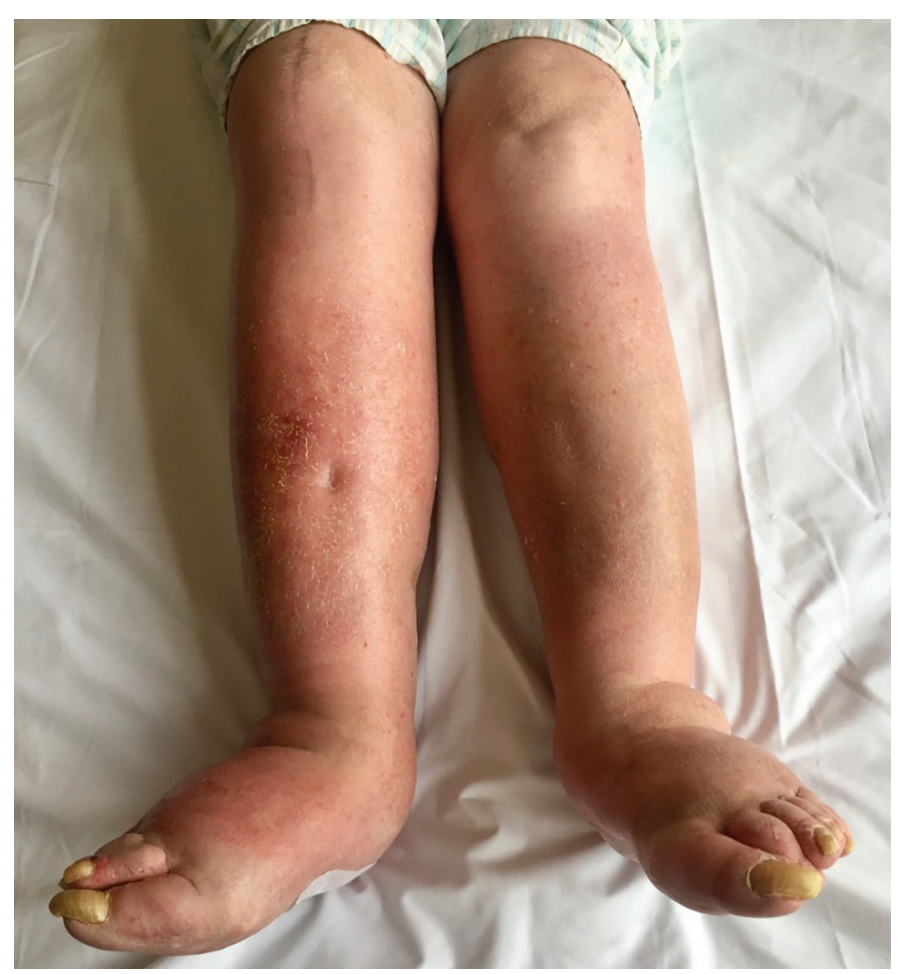

Figure 2 | Chronic pitting edema of the lower extremities in the same patient. The erythema on the right lower leg indicates incipient necrotizing cellulitis, a common complication of chronic edema.

elastic compression with reduced pressure and physiotherapy. After treatment, the patient exhibited significant edema reduction, wound healing, and a slight improvement in his general mobility. Compression was maintained with flat-knitted stockings. After the leg ulcer was healed (Fig. 3), low-dose methylprednisolone was continued and tocilizumab was not reintroduced.

\section{Discussion}

Pathophysiologically, edema is due to an imbalance between microvascular filtration and lymph drainage (2). Edema from too much capillary filtration is known as filtration edema or dynamic lymph insufficiency. Edema from reduced lymph transport is known as true lymphedema or static lymph insufficiency. A combination form of chronic peripheral edema is also possible. This terminology is not universally accepted, and some authors use the term lymphedema for all types of chronic edema. However, a distinction between the two pathophysiological types of edema is essential in order to determine optimal treatment $(2,3)$.

Chronic peripheral edema is a rare extra-articular feature of RA. It can appear on the lower and upper extremities. It does not seem to correlate with RF-positivity or with the clinical activity of the disease $(4,5)$. Pathophysiologically, chronic peripheral edema in RA patients can be either filtration edema or true lymphedema.

In the case presented here, frequent causes of filtration edema, such as heart, venous, and renal insufficiency, as well as hypoalbuminemia and secondary adrenal insufficiency after the cessation of long-term systemic corticosteroid treatment, were excluded. Myxoedema and secondary cutaneous amyloidosis, rare complications of RA, were also not confirmed. Due to the low $\mathrm{C}_{4}$ level, we considered type I acquired angioedema (AAE-I) in differential diagnosis. AAE-I has mainly been reported in patients with autoimmune diseases, such as systemic lupus erythematosus and eosinophilic granulomatosis (6-8). However, in this patient the quantitative and functional analyses of $\mathrm{C}_{1}$-inhibitor and $\mathrm{C}_{1 \mathrm{q}}$ levels were normal, and AAE was excluded from consideration.

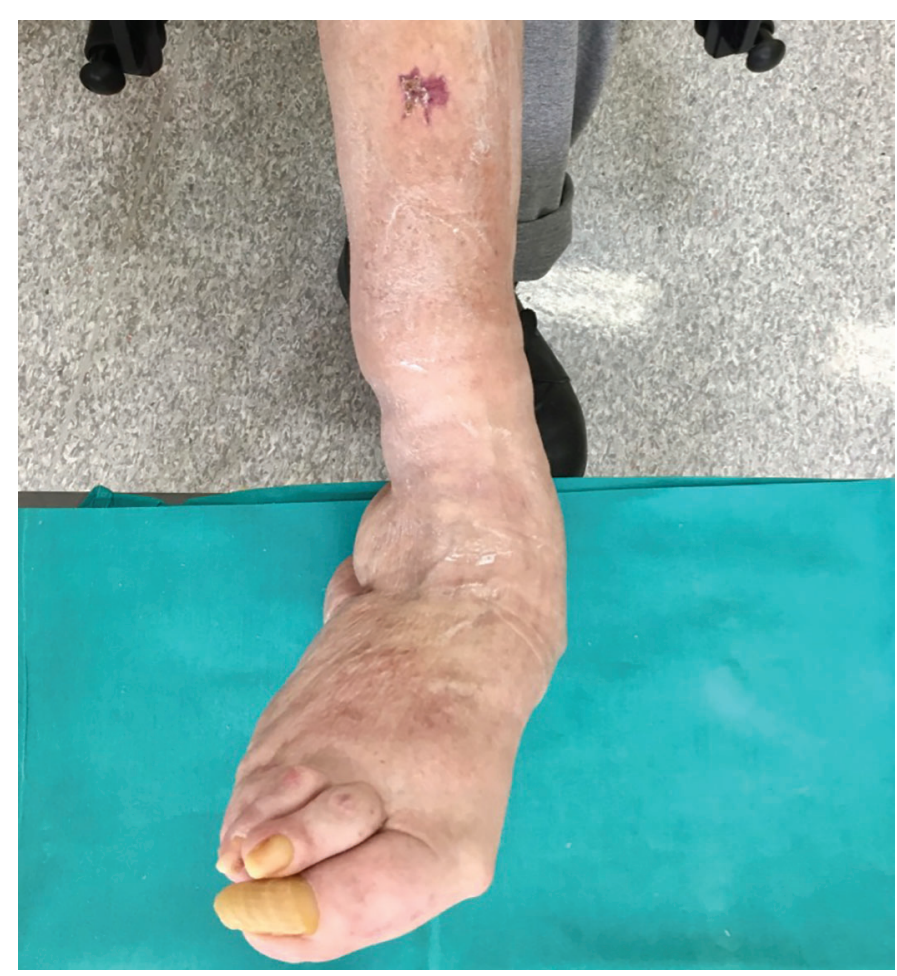

Figure 3 | A significant reduction of the edema and the fully healed ulcer from necrotizing cellulitis on the right lower extremity after modified intensive decongestive lymphatic therapy.

In patients with RA, filtration edema can be caused by the acute inflammation of synovia with excess lymph production $(4,5)$. That cause seemed unlikely in this patient due to the clinical and laboratory remission of RA. It is noteworthy that chronic peripheral edema started manifesting after treatment with tocilizumab, a humanized monoclonal antibody against the interleukin-6 receptor. Tocilizumab was discontinued in this patient because of a serious systemic infection: necrotizing cellulitis of the right lower leg. Peripheral edema with fluid retention is a rare reported side effect of tocilizumab $(9,10)$. Other medications the patient was taking-meloxicam, rosuvastatin, and metoprolol-are also known to cause chronic peripheral edema in $1 \%$ to $5 \%$ of patients and should be considered a potential cause of chronic peripheral edema (11). Immobility and leg dependency significantly increase capillary filtration due to gravity. At the same time, muscular inactivity causes the functional impairment of lymph flow because the lymphatic system requires external propulsion by means of tissue movement and deep breathing. Exercise can increase lymph transport capacity more than 10 -fold. Immobility results in minimal lymph drainage (3-5). This functional type of lymphatic impairment seems to be the most probable cause of chronic peripheral edema in the case described here.

True lyphedema in patients with RA is reported to be a consequence of the lymphatic obstruction by fibrin and other degradation products that accumulate in connective tissue and block the lymphatic channels (3). In the case described here, lymphoscintigraphy of the lower extremities revealed very slow lymphatic flow with no absolute obstruction. Thus, we have not directly proved lymphatic obstruction.

The cornerstone of lymphedema management is DLT, which consists of multilayer short stretch bandages, manual lymph drainage, skin treatment, and body exercises $(5,12-14)$. Due to poor mobility, the patient described in this case report received modified DLT with reduced pressure bandaging and regular physiotherapy without lymph drainage. This demonstrated that DLT is also efficient in patients with chronic peripheral edema and RA. 


\section{Conclusion}

It is of utmost importance to always consider a wide differential diagnosis when dealing with chronic peripheral edema in polymorbid patients. In the case presented here, the diagnosis established was of a combined form of chronic peripheral edema comprised of true lymphedema, which was confirmed by lymphoscintigraphy and could hypothetically be caused by the obstruction of lymph vessels due to the chronic inflammation of connective tissue, and filtration edema due to muscular inactivity, plus the suspected side effect of tocilizumab.

\section{References}

1. Moffat CJ, Franks PJ, Doherty DC, Williams AF, Badger C, Jeffs E, et al. Lymphoedema: an underestimated health problem. QJM. 2003;96:731-738.

2. Mortimer PS, Levick JR. Chronic peripheral oedema: the critical role of the lymphatic system. Clin Med Lond. 2004;4:448-453.

3. Foldi M, Foldi E. Foldi's textbook of lymphology. Mosby-Elsevier, 2006.

4. Minari C, Cecconami L, Fioravanti A, Montemerani M, Scola C, Marcolongo R. Lymphoedema of the limbs in rheumatoid arthritis. Clin Rheumatol. 1994;13:464-9.

5. Dacre JE, Scott DL, Huskisson EC. Lymphoedema of the limbs as an extra-articular feature of rheumatoid arthritis. Ann Rheum Dis. 1990;49:722-724.

6. Nettis E, Colanardi MC, Loria MP, Vacca A. Acquired C1-inhibitor deficiency in a patient with systemic lupus erythematosus: a case report and review of the literature. Eur J Clin Invest. 2005;35:781-4.

7. Ochonisky S, Intrator L, Wechsler J, Revuz J, Bagot M. Acquired $\mathrm{C}_{1}$ inhibitor deficiency revealing systemic lupus erythematosus. Dermatology. 1993;186:261-3.

8. Pasquali JL, Christmann D, Modert F, Belval PC, Storck D, Hauptmann G. First case of acquired functional $\mathrm{C}_{1}(-)$ INH deficiency: association with angioedema during Churg and Strauss vasculitis. Int Arch Allergy Appl Immunol. 1984;74:284-5.
9. eHealthMe. Actemra and Lymphedema - from FDA reports. c2017 [cited 2017 Sept 6]. Available from: http://www.ehealthme.com/ds/actemra/lymphoede$\mathrm{ma} /$.

10. Actemra (tocilizumab). Highlights of prescribing information. South San Francisco (CA): Genentech, Inc.; c2010 [cited 2017 Sept 6]. Available from https:// www.accessdata.fda.gov/drugsatfda_docs/label/2016/125276s107_125472so 18lbl.pdf.

11. Litt JZ. Litt's D.E.R.M. Drug eruptions \& reactions manual. 16th ed. Boca Raton, FL: CRC Press; 2010. p. 712.

12. International Lymphoedema Framework. Best practice for the management of lymphedema. International consensus. London: MEP Ltd. 2006;1-55.

13. Eyigor S, Karapolat H, Kirazli Y. Efficacy of etanercept and complete decongestive physical therapy in bilateral lower-limb lymphoedema associated with rheumatoid arthritis: a case report. Adv Ther. 2008;25:23-8.

14. International Society of Lymphoedema. The diagnosis and treatment of peripheral lymphedema: 2013 consensus document of the International Society of Lymphology. Lymphology. 2013;46:1-11. 